\title{
Ketonemia in dairy goats: Effect of dry period length and effect on lying behavior
}

\author{
G. Zobel, ${ }^{* 1}$ K. Leslie, $†$ D. M. Weary, ${ }^{*}$ and M. A. G. von Keyserlingk ${ }^{* 2}$ \\ *Animal Welfare Program, Faculty of Land and Food Systems, University of British Columbia, 2357 Main Mall, Vancouver, BC, \\ V6T 1Z4, Canada \\ †Department of Population Medicine, University of Guelph 2536 Stewart Building, Guelph, ON, N1G 2W1, Canada
}

\section{ABSTRACT}

In dairy animals, a successful transition from one lactation to the next includes minimizing negative energy balance. Cows experiencing excessive negative energy balance typically develop metabolic complications following parturition (e.g., ketosis); does are also susceptible before kidding (e.g., pregnancy toxemia). It is not known to what extent the provision and the length of the dry period affect these conditions in does. Furthermore, whereas clinical symptoms of these conditions include lethargy, behavioral changes resulting from ketosis and pregnancy toxemia have not been quantified in small ruminants. The aims of this study were to (1) describe the relationship between the dry period and negative energy balance, and (2) determine if lying behavior changes are indicative of the metabolic status of dairy goats. A total of 420 does on 10 commercial dairy goat farms in southern Ontario, Canada, were enrolled in the study (mean \pm SD: $42 \pm 18$ does/ farm). Each doe was affixed with a data logger to measure lying behavior from $12 \mathrm{~d}$ before to $12 \mathrm{~d}$ after kidding. Blood samples were collected at least once before and at least once following kidding to determine blood $\beta$-hydroxybutyrate (BHBA) concentration as an indicator of negative energy balance. Does were categorized as healthy (HLTH; both pre- and postkidding samples BHBA $<0.9 \mathrm{mmol} / \mathrm{L}$ ), PREGTOX (prekidding BHBA $\geq 1.7 \mathrm{mmol} / \mathrm{L}$ ), or KET (postkidding BHBA $\geq 1.7$ $\mathrm{mmol} / \mathrm{L})$. Behaviors were analyzed according to 5 periods: $\mathrm{P}-2$ ( $\mathrm{d}-12$ to $\mathrm{d}-2$ relative to kidding), $\mathrm{P}-1$ (d -1 relative to kidding), P0 (d 0, kidding day), P1 (d 1 relative to kidding), and $\mathrm{P} 2$ (d 2 to 12 relative to kidding). Dry period length and milk production after kidding were recorded when available. Farms ranged

\footnotetext{
Received November 21, 2014

Accepted May 4, 2015.

${ }^{1}$ Current address: AgResearch Limited, 10 Bisley Street, Private Bag 3123, Hamilton, New Zealand.

${ }^{2}$ Corresponding author: nina@mail.ubc.ca
}

from 0 to $15 \%$ and 0 to $50 \%$ in prekidding and postkidding ketonemia, respectively. The HLTH does had shorter dry periods compared with PREGTOX and KET does (43 vs. 55 d, SE of the differences of means $=4 \mathrm{~d}$ ). One farm kept some does milking, while drying off others; on this farm more PREGTOX and KET does $(11 / 28)$ were found in the dry group versus the continuously milked group $(1 / 16)$. Overall, does that had ketonemia before kidding (PREGTOX) spent more time lying down compared with healthy does (16.1 vs. $12.7 \mathrm{~h} / \mathrm{d}$, SE of the differences of means $=0.9$ ). Both PREGTOX and HLTH does had increased lying bouts in $\mathrm{P}-2$ compared with the day before $(\mathrm{P}-1)$ kidding [(mean (95\% CI): 16.8 (15.8-17.8) vs. 20.5 (19.4-21.8) bout/d]. Compared with HLTH does, animals that were ketonemic following kidding (KET) had higher mean lying times throughout the study (14.5 vs. 13.5 $\mathrm{h} / \mathrm{d}, \mathrm{SE}=0.4)$. Previous work has shown a relationship between shorter and skipped dry periods and improved energy balance in dairy cows; the current study is the first to indicate a similar relationship in dairy goats. Furthermore, goats that developed ketonemia, particularly before kidding, displayed decreased activity, including longer lying times and fewer lying bouts in the days around kidding.

Key words: dry off, metabolic disease, pregnancy toxemia, negative energy balance

\section{INTRODUCTION}

The transition between lactations is a challenging time for dairy animals in commercial production systems. Several management practices (e.g., feed changes, cessation of milking, and dry period length), as well as the physiological transitions between lactational and nonlactational states, and vice versa, have the potential to negatively affect welfare. A dry period is generally recognized as important for achieving optimal milk production (e.g., cows: Bachman and Schairer, 2003; ewes: Hernandez et al., 2012; does: Caja et al., 2006). The positive effects of the dry period on metabolic health are less certain. 
The end of the dry period and the beginning of the next lactation are frequently associated with a period of negative energy balance. This can be a consequence of increasing energy requirements of the growing fetus before parturition, as well as from the increasing metabolic demands of milk production after parturition. Although many animals are able to cope and recover from negative energy balance by metabolizing body fat, some animals metabolize too much fat, become overwhelmed with the associated by-products (e.g., NEFA and ketone bodies), and develop pregnancy toxemia (before parturition) and ketosis (following parturition). While ketosis may persist subclinically in cows, creating milk production losses (e.g., Rajala-Schultz et al., 1999; Goldhawk et al., 2009), pregnancy toxemia is often fatal in does and ewes if not diagnosed in a timely manner (e.g., Rook, 2000; Lima et al., 2012). In cows, the link between short or no dry periods and reduced risk of ketosis is clear (Rastani et al., 2005; de Feu et al., 2009). The metabolic health effect of carrying multiple fetuses (Forbes, 1968; Navarre and Pugh, 2002), and poor quality or restricted feed during the dry period (Laporte-Broux et al., 2011), are well known in does and ewes. However, the effect of dry period length has received little attention in these species.

Lethargy is commonly cited as a symptom of ketosis in cows, but evidence of reduced activity in sick animals has only recently been described (Itle et al., 2015). No similar evidence exists for does and ewes. Identifying and quantifying reduced activity could be particularly important in small ruminants because prognosis is poor by the stage at which clinical symptoms are documented. The majority of behavioral monitoring of goats to date has used either live observation (Loretz et al., 2004; Patt et al., 2013) or video (Loretz et al., 2004; Aschwanden et al., 2009). These methodologies have limited application in large commercial settings. Accelerometer-based data loggers are efficient in monitoring activity levels in cows (e.g., Ito et al., 2009; MedranoGalarza et al., 2012), but to date their application in goats has been minimal (e.g., Patt et al., 2012), and they have not been applied during kidding or to assess health status. Therefore, the study had 2 objectives. First, we sought to assess how does with pregnancy toxemia and ketosis vary with regard to provision of a dry period and dry period length. Second, we aimed to explore the merits of using changes in lying behavior (as assessed using data loggers) as early indicators of pregnancy toxemia and ketosis in dairy goats.

\section{MATERIALS AND METHODS}

This study was conducted in accordance to the University of British Columbia's Animal Care Certificate
A12-0249 and the University of Guelph's Animal Use Protocol 1636 as well as Behavioral Research Ethics Board at the University of British Columbia (H1202311) and the University of Guelph's Research Ethics Board (12NV014).

\section{Farms and Animals}

Ten commercial dairy goat farms in southern Ontario, Canada, participated in this trial. At the time of enrollment, farms were milking on average \pm SD $326 \pm 176$ does (range: 100-650 milking does). From these farms, a total of 420 multiparous, late gestation does (mean \pm SD: $42 \pm 18$ does/farm) were enrolled. Of these, 231 does were still milking and 189 does were already in the dry period. Most does were crossbred and were predominantly Saanen, Alpine, and La Mancha; one farm was composed entirely of the Saanen breed. Does were cared for according to each farm's established management protocols, and no changes to housing or general care were made by the research team. Feeding practices fell into 1 of 3 broad categories: (1) mixed forage supplemented with grain or concentrate, (2) TMR, or (3) complete pellet supplemented with straw, hay, or both. All farms dried off their does late in lactation, but one farm employed selective dry-off management, keeping high-producing does milking.

\section{Lying Behavior}

Approximately 2 wk before each doe's anticipated kidding date, a HOBO Pendant G data logger (Onset Computer Corporation, Bourne, MA) was attached vertically to a rear leg above the metatarsophalangeal joint using a self-adherent veterinary bandage (Vetrap, $3 \mathrm{M}$, St. Paul, MN) and foam pieces. Loggers were set to record at 1-min intervals. Loggers were removed and replaced every $21 \mathrm{~d}$ until approximately 2 wk of lying behavior before and after kidding was collected for each doe. Data were summarized according to the methodology outlined in Zobel et al. (2015) to calculate daily lying time and lying bout frequency for each doe. Early kiddings, doe deaths, logger failures, and lost loggers resulted in an average of $19 \pm 4 \mathrm{~d}$ of logger data per doe.

\section{Ketonemia}

Pregnancy toxemia and ketosis are associated with ketonemia, or elevated blood BHBA. The BHBA was measured using a Precision Xtra meter (Doré et al., 2013; Pichler et al., 2014) on blood samples collected via jugular venipuncture. Samples were collected at least once before kidding; if a doe did not kid within $14 \mathrm{~d}$ of 
her first sample, a second sample was taken. Samples used to establish prekidding BHBA concentration were collected on average $-7 \pm 4 \mathrm{~d}$ before kidding. The first postkidding sample was taken $9 \pm 4 \mathrm{~d}$ after kidding. Any doe with an elevated BHBA concentration (BHBA $=0.9-1.6 \mathrm{mmol} / \mathrm{L}$; Ramin et al., 2005) was flagged and sampled again every $5 \pm 2 \mathrm{~d}$ until BHBA either dropped below this threshold, or until it reached clinical concentrations (BHBA $\geq 1.7 \mathrm{mmol} / \mathrm{L})$. On average, these does with elevated BHBA concentrations were sampled 2.6 \pm 1.1 times. Prevalence of does that only had elevated BHBA concentrations $(\mathrm{BHBA}=0.9-1.6 \mathrm{mmol} / \mathrm{L} ; \mathrm{n}=$ 104) was calculated for descriptive purposes, but these does were not included in the final analysis. The final, complete data set contained the remaining does categorized into the following groups: HLTH (both pre- and postkidding samples BHBA $<0.9 \mathrm{mmol} / \mathrm{L} ; \mathrm{n}=243$ ), PREGTOX (prekidding BHBA $\geq 1.7 \mathrm{mmol} / \mathrm{L} ; \mathrm{n}=$ 15 , includes 2 does that had BHBA $\geq 1.7 \mathrm{mmol} / \mathrm{L}$ before and after kidding), and KET (postkidding BHBA $\geq 1.7 \mathrm{mmol} / \mathrm{L} ; \mathrm{n}=58)$. A total of 11 does died during the trial (PREGTOX $=7$ does, KET $=2$ does, and 2 does that died of other causes).

\section{Dry Period Length and Provision and Milk Production}

Dry period length was recorded for 227 does on 8 of the farms. Producers also provided the dry period length they intended to achieve. Milk production was recorded using computerized milking equipment matched with management software on 4 farms, and matched with manual, paper-based recording on 3 farms. One of these farms housed kids together with the does, resulting in low milk production after kidding. Three farms were not able to provide milk production data. Daily milk production for the first $2 \mathrm{wk}$ after kidding was used to calculate a single mean milk production value for each doe for that 2 -wk period.

\section{Statistical Analysis}

Feeding Practices. The complete data set [HLTH, $\mathrm{n}=243$; ketonemic (KETNMC), $\mathrm{n}=73$, combination of PREGTOX and KET] was used to test the effect of feeding practice on health status. A logistic regression (PROC GLIMMIX) with a binary distribution and a logit link function was used. Health status was the fixed effect, and doe nested within farm was the random effect. The number of kids a doe was carrying was included as a covariate. The logit-transformed results have been back-transformed and are presented as means and $95 \%$ confidence intervals, and include an odds ratio for the odds of a doe developing ketonemia either before or after kidding, based on the farm's feeding management practice.

Dry Period Length, Dry Period Provision, and Milk Production. Does with dry period length and milk production values were identified from the complete data set. This information was used to create a sub data set containing 147 does from 7 farms, where each doe had a dry period length and a health status (HLTH, $\mathrm{n}=119$; KETNMC, $\mathrm{n}=28$, combination of PREGTOX and KET). Complete milk production was missing for 8 does, resulting in a slightly smaller sub data set for milk production (HLTH, $\mathrm{n}=115$; KETNMC, $\mathrm{n}=24$, combination of PREGTOX and KET).

After removing all does that did not receive a dry period (e.g., dry period length of $0 \mathrm{~d}$; does that were continuously milked, either by accident on 2 farms or planned on one farm; $\mathrm{n}=19$ ), the relationship between health status (HLTH or KETNMC) and dry period length was assessed with a mixed model in SAS (PROC MIXED, SAS Institute Inc., Cary, NC). Health status was the fixed effect, and the farm's feeding practice and the number of kids a doe was carrying were included as covariates. Parity of does and milk production for the previous lactation were unavailable. Doe nested within farm was designated as the random effect. The residual term of the model was doe and the error term was doe nested within farm. The effect of health status (HLTH or KETNMC) on milk production following kidding was assessed using a similar model, except that the does that did not have a dry period $(n=19)$ were included, and that dry period length was also included as a covariate. The results are presented as means and standard error of the differences of the means, and line equations.

One farm from the above data set managed does on an individual animal level and opted to not provide a dry period when late lactation does were producing more than $1 \mathrm{~kg} / \mathrm{d}$. The does on this farm were given a binary categorization of their dry period status: 0 (no dry period, $\mathrm{n}=16$ ) or 1 (dry period, $\mathrm{n}=28$ ). To test the effect of provision of a dry period (continuous milking through to kidding vs. a dry period of any length) on health status (HLTH or KETNMC, combination of PREGTOX and KET), a 2-tailed Fisher's exact test was performed.

Lying Time and Lying Bout Frequency. After removing 11 does that did not have logger data, the HLTH does on all 10 farms $(n=232)$ were used in mixed models (PROC GLIMMIX, SAS 9.2) to test the fixed effect of day relative to kidding on lying time and lying bout frequency, with doe nested within farm as a random effect. A Gaussian distribution with identity 
link function was used for the lying time model, and a Poisson distribution with a log link function was used for the lying bout frequency model. Farm feeding practice and the number of kids a doe was carrying were included as covariates. Covariance structure autoregressive type 1 (AR1) was chosen for the lying time model, according to the smallest Akaike's information criterion, and covariance structure variance components was chosen based on a chi-square/df ratio close to 1.0 (lying bout frequency). Least squares means and SEM were plotted for lying time by day relative to kidding and least squares means and $95 \%$ confidence interval were plotted for lying bouts by day relative to kidding; based on these plots, contrast statements were written to test the mean differences between day of interest surrounding kidding ( $\mathrm{d}-2,-1,0,1$, and 2), and 5 periods were then identified for use in the health status models: $\mathbf{P} \mathbf{- 2}$ (d -12 to -2 relative to kidding), $\mathbf{P}-\mathbf{1}$ (d -1 relative to kidding), $\mathbf{P 0}$ (d 0, kidding day), $\mathbf{P} 1$ (d 1 relative to kidding), and $\mathbf{P 2}$ (d 2 to 12 relative to kidding).

Separate data sets were created for testing health status and lying behavior because it was anticipated that differences would exist between does that developed ketonemia before kidding (PREGTOX) and does that developed ketonemia following kidding (KET). Five does with insufficient data logger data were removed (1 PREGTOX doe died with only $1 \mathrm{~d}$ of data and $4 \mathrm{KET}$ does had failed or missing loggers). The final PREGTOX data set contained 14 does that were ketonemic before kidding (PREGTOX) and $232 \mathrm{HLTH}$ does. The final KET data set contained 54 does that were ketonemic after kidding (KET) and $232 \mathrm{HLTH}$ does.

For both data sets, mixed models (PROC GLIMMIX, SAS 9.2) were used to assess the effect of health status (HLTH vs. either PREGTOX or KET, depending on the data set) on lying time (Gaussian distribution with identity link function) and lying bout frequency (Poisson distribution with a log link function). Health status was the fixed effect, and doe nested within farm and period nested within doe were random effects. Farm feeding practice and the number of kids a doe was carrying were included as covariates. An interaction between health status and period was tested. Differences between health status on each period were tested post-hoc using Dunnett's 2-tailed tests. For lying time, results are presented as means and $\mathrm{SE}$ and line equations. For lying bout frequency, the logit-transformed results have been back-transformed and are presented as means and $95 \%$ confidence intervals. The residual term of the models was period nested within doe, and the error term to test the effect of health status was doe nested within farm. Based on the best-fit statistics, auto-regressive type 1 (AR1) covariance structure was selected for KET models and variance components covariance structure was selected for PREGTOX models.

For all models, residuals were calculated and examined for normality and homogeneity of variances. Possible outliers were also identified using residuals; data points that were 3 or more times the interquartile range away from the first and third quartile were considered outliers. Based on this method, the HLTH data set (n $=232$ ), used to illustrate typical doe lying behavior around kidding, had 11 outliers removed ( 7 from the lying time model, and 4 from lying bout frequency model). The KET data set had 1 outlier removed from the lying time model.

\section{RESULTS}

\section{Prevalence of Ketonemia}

All but one farm had some prevalence $(22 \pm 13 \%$, range $=0-48 \%$ ) of elevated BHBA concentrations $(\mathrm{BHBA}=0.9-1.6 \mathrm{mmol} / \mathrm{L})$. In regards to ketonemia (BHBA $\geq 1.7 \mathrm{mmol} / \mathrm{L})$, some farms had low prevalence of ketonemia before kidding ( $\mathrm{n}=5$ farms; PREGTOX mean \pm SD: $4 \pm 6 \%$, range $=0-15 \%)$ and ketonemia after kidding ( $\mathrm{n}=8$ farms; KET mean \pm SD: $14 \pm$ $15 \%$, range $=0-50 \%)$. One farm avoided all issues, with no elevated BHBA concentrations, and no ketonemia before and after kidding. Overall, ketonemia following kidding (KET, $\mathrm{n}=58$ does) was more prevalent than before kidding (PREGTOX, $\mathrm{n}=15$ does). Figure 1 illustrates the prevalence of PREGTOX and KET across farms grouped according to feeding practice. Both the number of kids a doe was carrying and the farm's feeding practice affected health status. The odds of does becoming ketonemic (PREGTOX and KET combined) when carrying triplets compared with singles or twins were greater by a factor of 2.4 (95\% CI: $1.1-5.2 ; P$ $<0.05$ ), whereas the odds of does becoming ketonemic (PREGTOX or KET combined) on farms feeding a complete pellet diet compared with those feeding a total mixed ration or forage supplemented with concentrate were greater by a factor of 4.5 (95\% CI: $1.0-20.1$; $P<0.05)$.

\section{Dry Period Length, Provision of a Dry Period, and Milk Production}

All but one farm indicated they aimed for a $60-\mathrm{d}$ dry period. The observed dry period ranged from 0 to 109 d dry (mean \pm SD: $40 \pm 22$ d). Excluding the does that were not provided a dry period, does that 


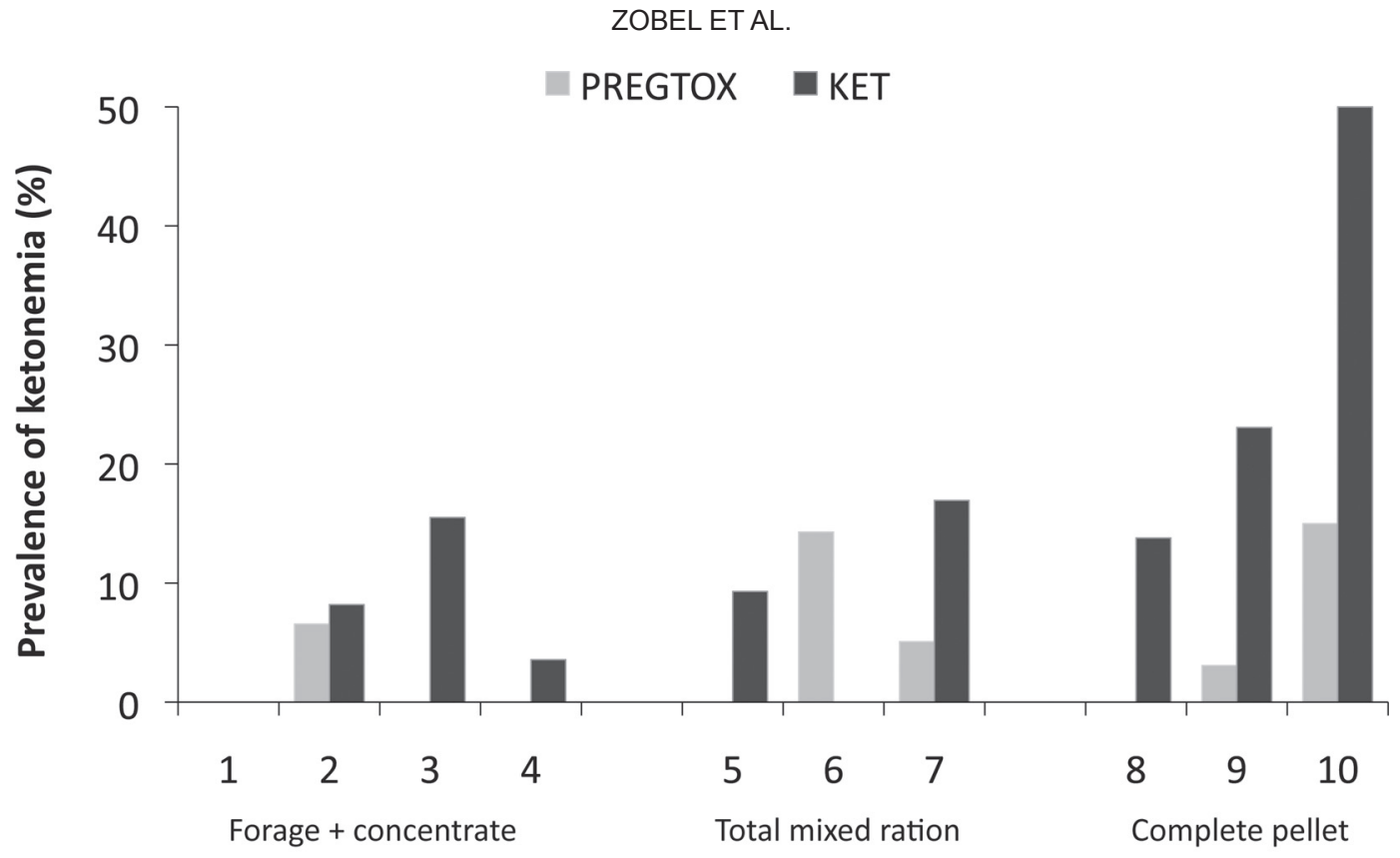

Farm no. (grouped by feeding practice)

Figure 1. Prevalence of ketonemia (blood BHBA $\geq 1.7 \mathrm{mmol} / \mathrm{L})$ before kidding (PREGTOX; $\mathrm{n}=15)$ or after kidding $($ KET; $\mathrm{n}=58)$ on 10 commercial dairy farms (total does enrolled $=420)$. Does with blood BHBA $<0.9 \mathrm{mmol} / \mathrm{L}$ classified as HLTH $(\mathrm{n}=243)$ and those with elevated concentrations (blood BHBA 0.9-1.6 mmol/L; $\mathrm{n}=104$ ) are not shown on the graph.

stayed healthy before and after kidding had shorter dry periods compared with does that developed ketonemia (PREGTOX and KET combined; 43 vs. $55 \pm 4 \mathrm{~d} ; P$ $<0.05)$. The type of feed a farm was feeding and the number of kids a doe was carrying did not influence the model $(P>0.10)$. On the farm that opted to keep some of its does milking, while giving other does dry periods, more ketonemia (PREGTOX and KET combined) was found in the dried-off animals (11/28 does) compared with those that were kept milking $(1 / 16$ does; Fisher's exact test, $P<0.05)$.

Milk production was negatively affected by health status, the type of feed a farm was feeding, and the number of days in the dry period. The overall line equation for this model was milk production $(\mathrm{kg} / \mathrm{d})=-(0.5$ $\times$ ketonemic $)-(0.7 \times$ complete pellet feeding $)-(0.01$ $\mathrm{d}$ in dry period $)+3.8(P<0.05)$. The number of kids a doe was carrying did not affect milk production in the first 2 wk of lactation $(P>0.10)$.

\section{Lying Behavior}

The HLTH does on all 10 farms maintained their lying time until $\mathrm{d}-1$, at which point they spent less time lying. Two days after kidding, they returned to their prekidding levels (Figure 2). On average, healthy does carrying triplets $(n=26)$ lay longer than healthy does carrying singles $(\mathrm{n}=68)$ or twins $(\mathrm{n}=138)(15.1$ vs. $14.1 \mathrm{~h} / \mathrm{d}, \mathrm{SE}$ of the differences of means $=0.9 ; P$ $<0.05)$. Lying bouts increased as does neared kidding (lying bouts $/ \mathrm{d}=0.36 \mathrm{~d}+19.5 ; P<0.05)$, with the greatest increases on $d-1$ and 0 (Figure 3 ). The number of kids a doe was carrying had no effect on lying bouts. The type of feed a farm fed did not affect either lying time or lying bout frequency of healthy does.

In does that became ketonemic before kidding (PREGTOX), the lying times were different overall (16.1 vs. $12.7 \mathrm{~h} / \mathrm{d}, \mathrm{SE}=0.9 ; P<0.05)$ compared with HLTH does. The PREGTOX does spent more time lying down during all periods compared with HLTH does (Table 1). Does carrying triplets tended to have longer lying times by $1.0 \mathrm{~h} / \mathrm{d}(P<0.10)$. The type of feed farms were feeding did not have an effect on the model. No interaction was found between period relative to kidding and health status in terms of lying time. Furthermore, although PREGTOX and HLTH does were not different in the frequency of daily lying bouts before kidding, all does increased the number of lying bouts between $\mathrm{P}-2$ and $\mathrm{P}-1$ [ $($ mean $(95 \% \mathrm{CI}): 16.8$ (15.8-17.8) vs. $20.5(19.4-21.8)$ bout/d; $P<0.0001)]$. The number of kids a doe was carrying did not affect lying bouts $(P>0.10)$.

In does that developed ketonemia after kidding (KET), overall lying time was higher compared with 


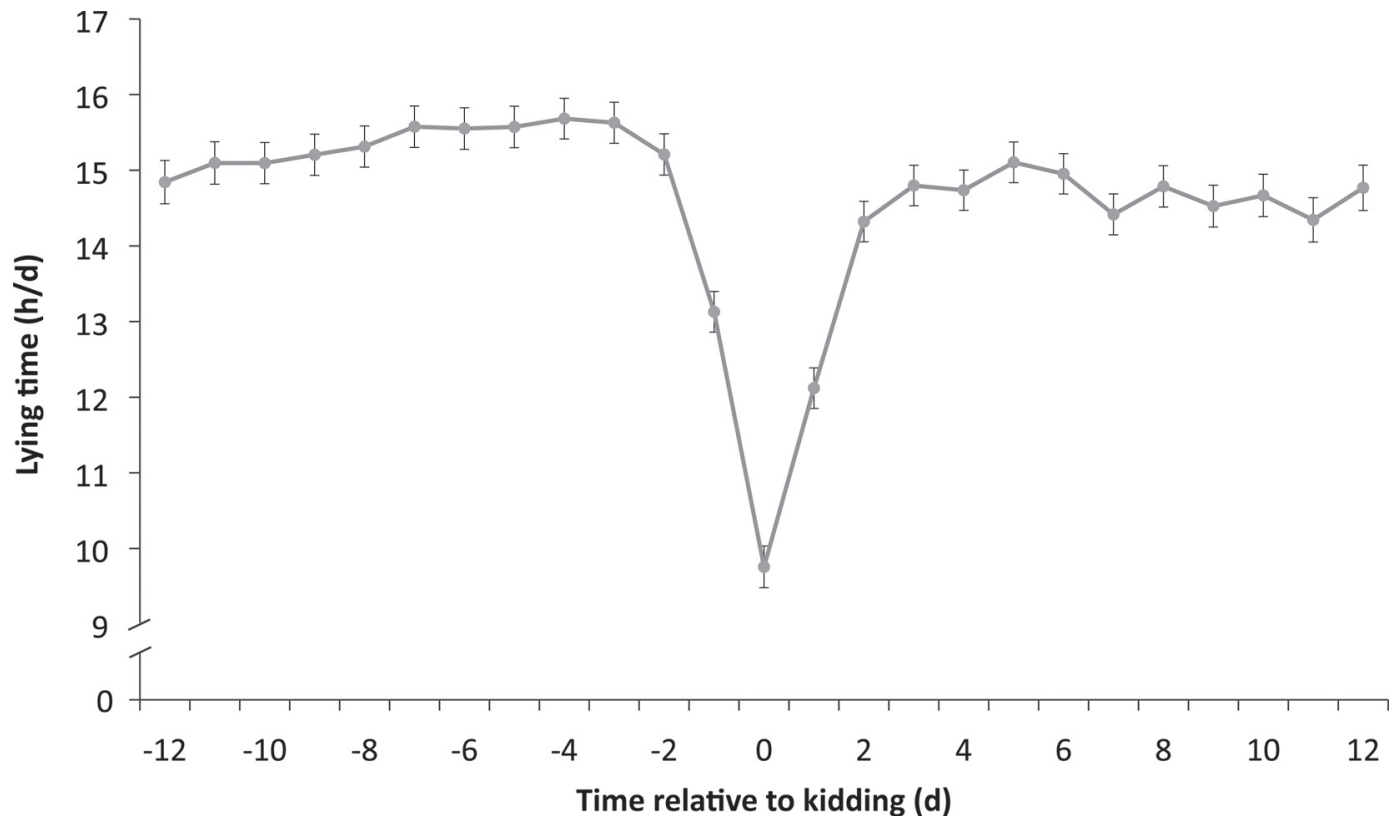

Figure 2. Daily lying time for $12 \mathrm{~d}$ before and $12 \mathrm{~d}$ following kidding for healthy does with lying behavior data from all 10 farms (no ketonemia for the entire study; blood BHBA $<0.9 \mathrm{mmol} / \mathrm{L} ; \mathrm{n}=232$ ). The standard error of the mean is from the model testing effect of day relative to kidding on daily lying time.

does that stayed healthy throughout the study $(14.5$ health status $(P<0.05)$, with KET does lying down vs. $13.5 \mathrm{~h} / \mathrm{d}$, SE of the differences of means $=0.4 ; P$ more than HLTH does on the days around kidding $<0.05)$. An interaction was found between period and ( $\mathrm{P}-1, \mathrm{P} 0$, and $\mathrm{P} 1$; Table 2). Does that were carry-

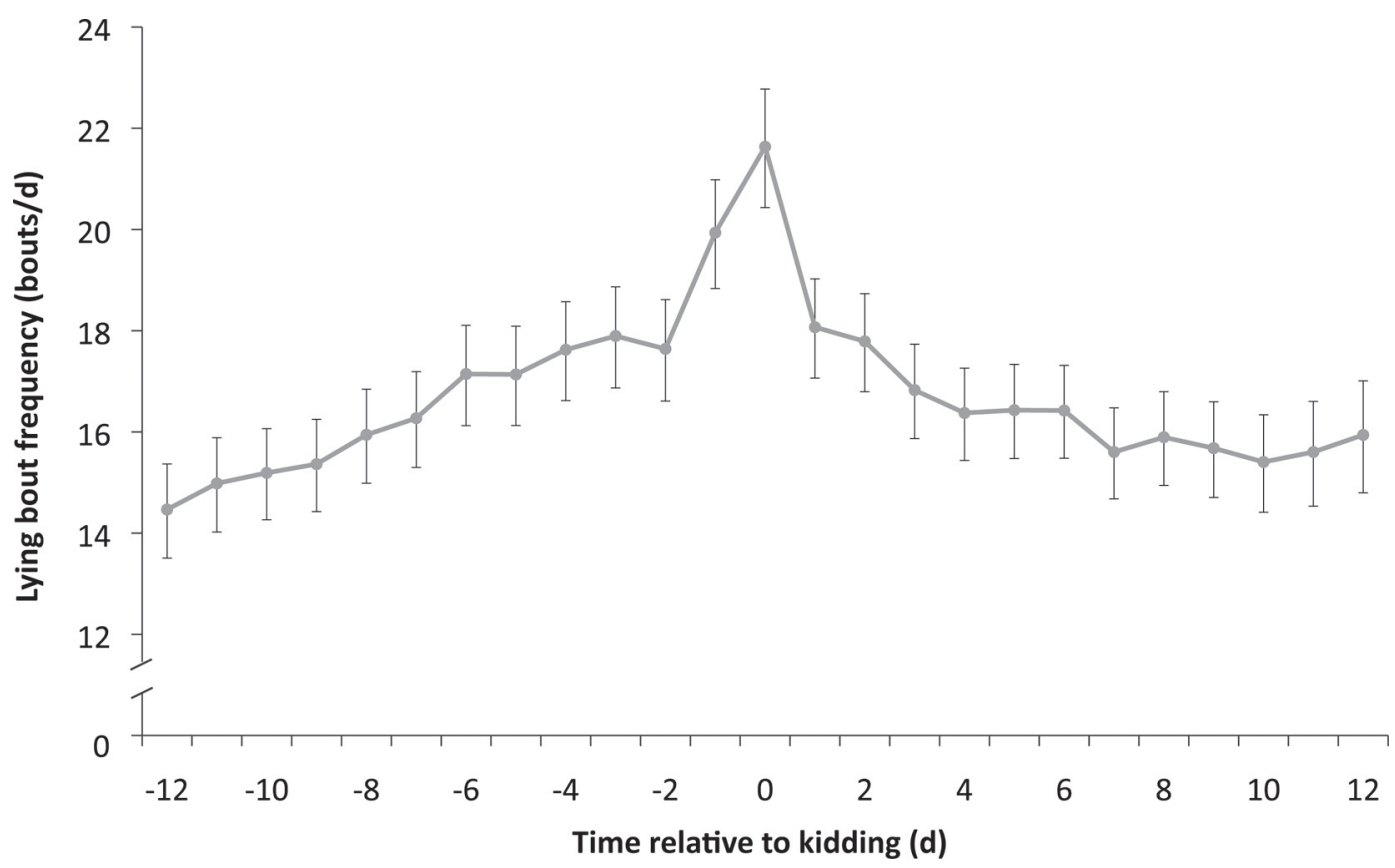

Figure 3. Daily lying bout frequency for $12 \mathrm{~d}$ before and $12 \mathrm{~d}$ following kidding for healthy does with lying behavior data from all 10 farms (no ketonemia for the entire study; blood BHBA $<0.9 \mathrm{mmol} / \mathrm{L} ; \mathrm{n}=232$ ). Error bars represent upper and lower $95 \%$ confidence interval from the model testing effect of day relative to kidding on daily lying bout frequency. 
Table 1. Mean daily lying time of does with ketonemia (blood BHBA $\geq 1.7 \mathrm{mmol} / \mathrm{L}$ ) before kidding (PREGTOX) is compared with daily lying time of does that maintained normal levels (blood BHBA $<0.9$ $\mathrm{mmol} / \mathrm{L})$ for the entire study $(\mathrm{HLTH})^{1}$

\begin{tabular}{|c|c|c|c|c|}
\hline \multirow{2}{*}{$\begin{array}{l}\text { Period relative } \\
\text { to kidding }{ }^{2}\end{array}$} & PREGTOX $(\mathrm{n}=14)$ & HLTH $(\mathrm{n}=232)$ & \multirow[b]{2}{*}{ SED } & \multirow[b]{2}{*}{$P$-value } \\
\hline & Lying time $(\mathrm{h} / \mathrm{d})$ & Lying time $(\mathrm{h} / \mathrm{d})$ & & \\
\hline-2 & 17.7 & 15.4 & 1.0 & $<0.05$ \\
\hline-1 & 16.0 & 13.2 & 1.1 & $<0.05$ \\
\hline 0 & 13.0 & 9.8 & 1.1 & $<0.01$ \\
\hline
\end{tabular}

${ }^{1}$ Due to doe deaths, the comparison was only possible for the first 3 periods relative to kidding. Data are from all 10 farms. SED = standard error of the differences of means.

${ }^{2}$ Period $-2=$ mean of $\mathrm{d}-12$ to -2 relative to kidding; period $-1=\mathrm{d}-1$ relative to kidding; and period $0=$ d 0 , kidding day.

ing triplets lay down $1.3 \mathrm{~h} / \mathrm{d}$ more than those carrying singles or twins $(P<0.05)$. The farm's feeding practice also affected the model, with does on complete pellet feeds lying almost an hour longer compared with does on farms feeding TMR or forage supplemented with concentrate $(P<0.05)$. With regard to lying bout frequency, KET does had a tendency to get up and down less frequently than HLTH does during $\mathrm{P}-2$ (mean and $95 \% \mathrm{CI}): 14.8(13.2-16.6)$ vs. $16.8(15.9-17.7 ; P<0.10)$ and $\mathrm{P}-1$ (mean and $95 \% \mathrm{CI}$ ): $18.3(16.2-20.6)$ vs. 20.4 $(19.3-21.6 ; P<0.10)$. The farm's feeding type and the number of kids a doe was carrying did not affect lying bouts $(P>0.10)$.

\section{DISCUSSION}

Pregnancy toxemia and ketosis have a variety of physical signs, such as ataxia, reduced activity levels, teeth grinding, and anorexia (Menzies and Bailey, 1997; Brozos et al., 2011; Lima et al., 2012). However, these signs are often associated with other diseases, making blood and urine ketone concentrations (e.g., as indicated by elevated BHBA; ketonemia and ketonuria, respectively) helpful in reaching a diagnosis. This study measured ketonemia using on-farm blood testing. Some have debated the precise concentrations of BHBA that should be considered diagnostic. For example, Marteniuk and Herdt (1988) defined severe pregnancy toxemia and ketosis using BHBA $>3.0 \mathrm{mmol} / \mathrm{L}$. Ramin et al. (2005) used blood BHBA concentrations between 0.8 and $1.6 \mathrm{mmol} / \mathrm{L}$ as indicative of subclinical pregnancy toxemia. Ismail et al. (2008) used $0.86 \mathrm{mmol} / \mathrm{L}$ as a threshold for subclinical pregnancy toxemia. In the current study, does that died had a mean blood BHBA of $5.0 \mathrm{mmol} / \mathrm{L}$ and ranged from 1.7 to $6.9 \mathrm{mmol} / \mathrm{L}$. Given this combined evidence, we used a cutoff of $1.7 \mathrm{mmol} / \mathrm{L}$ to define both pregnancy toxemia and ketosis. One limitation of the current study was the inability to continue testing to determine how long BHBA remained above $1.7 \mathrm{mmol} / \mathrm{L}$. We encourage future research to use more frequent and longer-term sampling.

On one farm, none of the does sampled had ketonemia or even elevated blood BHBA concentrations. This farm had experienced cases of pregnancy toxemia in the past and had developed a protocol for managing late gestation does, including providing supplemental concentrate to does carrying multiple kids, feeding several small meals throughout the day, and feeding highly palatable forage. It is unclear which, if any, of these changes were responsible for the excellent health status

Table 2. Mean daily lying time of does with ketonemia (blood BHBA $\geq 1.7 \mathrm{mmol} / \mathrm{L}$ ) after kidding (KET) is presented and compared over 5 periods to the lying time of does that maintained normal concentrations (blood BHBA $<0.9 \mathrm{mmol} / \mathrm{L})$ for the entire study $(\mathrm{HLTH})$

\begin{tabular}{lccccc}
\hline & KET $(\mathrm{n}=54)$ & & HLTH $(\mathrm{n}=232)$ & & \\
\cline { 2 - 2 } $\begin{array}{l}\text { Period relative } \\
\text { to kidding }\end{array}$ & Lying time $(\mathrm{h} / \mathrm{d})$ & & Lying time $(\mathrm{h} / \mathrm{d})$ & SED $^{2}$ & P-value \\
\hline-2 & 16.2 & 15.7 & 0.5 & $>0.10$ \\
-1 & 14.7 & 13.5 & 0.5 & $<0.05$ \\
0 & 12.2 & 10.2 & 0.6 & $<0.01$ \\
1 & 13.7 & 12.5 & 0.6 & $<0.05$ \\
2 & 15.5 & 15.2 & 0.5 & $>0.10$ \\
\hline
\end{tabular}

${ }^{1}$ Period $-2=$ mean of $\mathrm{d}-12$ to -2 relative to kidding; period $-1=\mathrm{d}-1$ relative to kidding; period $0=\mathrm{d} 0$, kidding day; period $1=\mathrm{d} 1$ relative to kidding; and period $2=$ mean of $\mathrm{d} 2$ to $\mathrm{d} 12$ relative to kidding.

${ }^{2} \mathrm{SED}=$ standard error of the differences of means. 
of does on this farm. Overall, the prevalence of ketonemia before kidding in this study was low, with only half of the farms having any cases; this result is consistent with previous studies (e.g., Melby et al., 1986). Unfortunately, mortality associated with the cases found in our study was very high (7 out of 15 does died), which is again consistent with previous literature (Malher et al., 2001; Lima et al., 2012).

In the current study, all but one farm had higher prevalence of ketosis than pregnancy toxemia. This outcome was unanticipated because veterinary resources (e.g., Andrews, 1997; Edmondson and Pugh, 2004; Brozos et al., 2011) typically focus on pregnancy toxemia, and metabolic issues following kidding are less discussed. Because the latter condition is linked to the increasing metabolic demands of milk production (e.g., Goldhawk et al., 2009), and improvements in nutritional formulations are resulting in rapidly increasing milk production in dairy goats (e.g., Monzón-Gil et al., 2010; Goetsch et al., 2011), we suggest that ketosis will be a growing issue in dairy goats.

The present study found reduced milk production in does that had ketonemia before or after kidding, which is similar to the work showing milk production losses in cows (e.g., Rajala-Schultz et al., 1999; Goldhawk et al., 2009). These losses, coupled with treatment costs (Guard, 1994), have motivated a large body of literature examining this disease in cows. One emerging discussion centers on reducing ketosis by manipulating dry period length. Although not all studies agree (see review by van Knegsel et al., 2013), decreasing or skipping the dry period may assist in preventing ketosis in dairy cows after calving. For instance, de Feu et al. (2009) found that cows provided no dry period had increased energy balance and better body condition compared with cows provided a standard (60 d) dry period. Several factors contribute to such improvements, including the feed management necessitated by continued lactation. Cows with shorter or skipped dry periods must be fed a higher energy diet to support milk production, and when this is done, cows maintain higher DMI and mobilize less fat reserves postpartum, ultimately resulting in improved energy balance (Rastani et al., 2005). These metabolic benefits must be balanced with possible decreased milk production in the next lactation; however, this seems to be more of any issue when the dry period is skipped entirely (Rastani et al., 2005). Indeed, Jolicoeur et al. (2014) found that reducing the dry period length increased DMI in the next lactation, improving metabolic status without negatively affecting milk production. In the current study, longer dry periods were linked to reduced milk production. Further, does that remained healthy had shorter or skipped dry periods. Unfortunately, this does not indicate that managing does for shorter dry periods actually reduces the risk of pregnancy toxemia and ketosis given the possible confound between does self-drying and ketonemia. Although all but one farm indicated they managed their does based on a fixed number of days until kidding (e.g., $60 \mathrm{~d}$ dry), the large range in dry periods observed indicates that the majority of these farms actually dried off does based on their milk production, meaning that the actual dry period of the does was influenced by milk production persistency. Although the relationship between milk production persistency and metabolic health status are not well understood, possible benefits of continued production were observed on one farm where does were kept milking if they continued to produce more than $1 \mathrm{~kg} / \mathrm{d}$. It can be argued that healthier does will continue to produce more milk than does that are already compromised. Thus, it is possible that does with longer dry periods were already metabolically challenged or at a higher risk of illness. Randomly assigning dry period in a controlled trial, using dairy goats where parity and the previous lactation's milk production is known (unlike in this study) is necessary to disentangle the effects of dry period length on metabolic function (similar to studies on cows; Rastani et al., 2005). However, as demonstrated by Caja et al. (2006), assigning shorter dry periods may be difficult as lactation persistence varies in does, resulting in some spontaneous self-drying.

Two other factors noted for their influence in challenging goats metabolically around kidding are kid numbers and feeding regimen. The number of kids a doe is carrying is pertinent because multiple fetuses reduce rumen capacity (Forbes, 1968), limiting feed intake ability and thus promoting negative energy balance. Energy requirements in late gestation can more than double in ewes carrying twins and triplets compared with those carrying singles (Navarre and Pugh, 2002). Schlumbohm and Harmeyer (2008) showed that twins increased the susceptibility of ewes to pregnancy toxemia. Similarly, Lima et al. (2012) found that does with pregnancy toxemia carried more kids compared with controls ( 2.7 vs. 2.0 kids). In the current study, we found a similar pattern with greater odds of ketonemia in does carrying triplets.

The second factor with potential to affect energy balance is feeding regimen. Our results showed that almost half of all the cases of ketosis were observed on the 3 farms feeding a "complete pellet" supplemented with straw and sometimes hay. We also observed that does on these farms were at increased risk of becoming ketonemic. These results should be viewed with caution 
given that the purpose of the study was not to evaluate the effect of feeding practices on health status. However, the findings do provide some evidence that more insight is needed regarding the effects of concentrate and forage type (e.g., pelleted vs. textured feed) on ketonemia in does. Further work to investigate feed management practices for avoiding negative energy balance in early lactation is encouraged.

Once pregnancy toxemia is diagnosed, the prognosis is often poor (Lima et al., 2012). This situation was reflected in the protocols put in place by the farms with which we worked. Only a few of the producers attempted to treat does after the animals began to show clinical symptoms. On one farm, the protocol was to euthanize the dam, and perform a cesarean section to save the kids. Lima et al. (2012) used several treatment protocols, alone and in combination [i.e., performing a cesarean section; oral administration of a commercially available electrolyte product or propylene glycol (or both); intravenous administration of a glucose solution or bicarbonate solution (or both), and in does with swollen joints, flunixin meglumine; and subcutaneous administration of calcium borogluconate solution]. However, of the 22 clinical cases reported in the Lima et al. (2012) study, 19 does died, so the efficacy of these treatments cannot be established. With earlier diagnosis of sick or at risk animals, it may be possible to develop more effective treatment protocols.

Using lying behavior for identifying health issues in cows has become increasingly popular with the advent of accelerometer-based monitoring devices. These monitors have been used to evaluate lying behavior (Ito et al., 2009), mastitis (Medrano-Galarza et al., 2012), lameness (Ito et al., 2010), and clinical ketosis (Itle et al., 2015). The current study found that these devices are useful for identifying behavioral changes in dairy goats. Healthy does showed increases in activity nearing parturition, and dramatic drops in lying times in the day before, day of, and day after kidding. Similar patterns have been noted in cows (Huzzey et al., 2005). Although the ketonemic does in the present study displayed similar patterns, the changes were not as pronounced as in the healthy does. They generally spent longer lying down in the days before kidding compared with their healthy counterparts; this difference was especially obvious in the does with ketonemia before kidding. Because our monitoring period only collected data $12 \mathrm{~d}$ before kidding, future work should monitor does even earlier to identify when lying time changes begin.

All does reduced lying time on the day of kidding, but the ketonemic does did not have as dramatic a decrease as does that stayed healthy. These results con- trast those of Itle et al. (2015), who found that severely ketotic cows stood more than healthy cows. Itle et al. (2015) also found that ketotic cows did not increase their activity level on the day of calving, whereas in the present study ketonemic does had the same number of lying bouts as healthy does. In fact, the number of times does got up and down was lower overall compared with healthy does, which was not the case for Itle et al. (2015). In the latter study, cows were moved to a calving pen when parturition was imminent, whereas in the current study does remained in a group pen until after kidding. It is possible that the lack of pen movement in the present study resulted in does feeling comfortable to stay lying down for longer, whereas the cows in Itle et al. (2015) study were faced with a new environment that is known to result in restlessness and more standing bouts (Proudfoot et al., 2013).

Most of the lying behavior differences in this study were more evident for does that were ketonemic before kidding. We may have been better able to detect health effects prekidding because management at this time was more similar among farms than it was after kidding. For instance, all of the farms housed prekidding does together in groups, and new does were not typically added to these groups. Introducing new individuals into a group of goats has been shown to disturb normal behavior (Patt et al., 2012). Further, although stocking density was not recorded, all does were able to lie down with minimal disturbance in the kidding areas. Andersen and Bøe (2007) showed that goats spend more time resting simultaneously when provided more space. In comparison, both the group dynamics and the space provision for goats after kidding were more variable among the farms. The lying time of the healthy does from all 10 farms corresponded to this, with very consistent daily time spent lying before kidding, and more variable lying time in the days afterward. Patt et al. (2012) found that goats tended to self-isolate when moved into a new group, increasing their lying time. It is possible that because of their move into a lactating pen, both ketonemic and healthy does were behaving similarly with regard to lying time. Furthermore, because introduction into a new group is associated with reduced time spent feeding (Patt et al., 2012), we suggest that the high prevalence of ketonemia after kidding in this study may be in part due to a change in feeding behavior. Further work examining the feeding behavior of does directly after kidding in more controlled settings is encouraged. Finally, because low ranking (Andersen and Bøe, 2007) and younger (Szabò et al., 2013) does tend to be most affected by constraints and changes in their environment, future work should consider social status and age. 


\section{CONCLUSIONS}

Ketonemia following kidding (i.e., ketosis) was more prevalent than ketonemia before kidding (i.e., pregnancy toxemia). Does that remained healthy before and after kidding had shorter dry periods. When does were managed to have no dry period, more animals remained healthy compared with those that were dried off. Reducing or eliminating dry periods in dairy goats is a promising management practice; however, further work is needed to disentangle the effect impending illness may have played on lengthening the dry period regardless of management. Does with pregnancy toxemia had higher lying times before kidding. Does with ketosis showed decreased lying times on the day before, the day of, and day after kidding only. These results suggest that automated measures of lying behavior are a promising indicator of pregnancy toxemia and ketosis.

\section{ACKNOWLEDGMENTS}

The authors thank Mark Wynands (University of Guelph), the staff at the Department of Population Medicine, University of Guelph (Guelph, ON), and the staff at Ontario Goat (Guelph, ON). The project was funded by a grant provided by the Ontario Centre of Excellence in Goat Research and Innovation (Peterborough, Ontario, Canada). Gosia Zobel was supported by Canada's Natural Science and Engineering Research Council's PGSD (Postgraduate Scholarships-Doctoral) Scholarship (Ottawa, ON, Canada).

\section{REFERENCES}

Andersen, I. L., and K. E. Bøe. 2007. Resting pattern and social interactions in goats - The impact of size and organisation of lying space. Appl. Anim. Behav. Sci. 108:89-103.

Andrews, A. 1997. Pregnancy toxaemia in the ewe. In Pract. 19:306314 .

Aschwanden, J., L. Gygax, B. Wechsler, and N. M. Keil. 2009. Loose housing of small goat groups: Influence of visual cover and elevated levels on feeding, resting and agonistic behaviour. Appl. Anim. Behav. Sci. 119:171-179.

Bachman, K. C., and M. L. Schairer. 2003. Invited review: Bovine studies on optimal lengths of dry periods. J. Dairy Sci. 86:30273037.

Brozos, C., V. S. Mavrogianni, and G. C. Fthenakis. 2011. Treatment and control of peri-parturient metabolic diseases: Pregnancy toxemia, hypocalcemia, hypomagnesemia. Vet. Clin. North Am. Food Anim. Pract. 27:105-113.

Caja, G., A. A. K. Salama, and X. Such. 2006. Omitting the dry-off period negatively affects colostrum and milk yield in dairy goats. J. Dairy Sci. 89:4220-4228.

de Feu, M. A., A. C. O. Evans, P. Lonergan, and S. T. Butler. 2009. The effect of dry period duration and dietary energy density on milk production, bioenergetic status, and postpartum ovarian function in Holstein-Friesian dairy cows. J. Dairy Sci. 92:6011-6022.

Doré, V., J. Dubuc, A. M. Bélanger, and S. Buczinski. 2013. Short communication: Evaluation of the accuracy of an electronic onfarm test to quantify blood $\beta$-hydroxybutyrate concentration in dairy goats. J. Dairy Sci. 96:4505-4507.
Edmondson, M. A., and D. G. Pugh. 2004. Pregnancy toxemia in sheep and goats. Pages $144-145$ in Current Veterinary Therapy: Food Animal Practice. D. E. Anderson and D. M. Rings, ed. Saunders Elsevier, St. Louis, MO.

Forbes, J. M. 1968. The physical relationship of the abdominal organs in the pregnant ewe. J. Agric. Sci. 70:171-177.

Goetsch, A. L., S. S. Zeng, and T. A. Gipson. 2011. Factors affecting goat milk production and quality. Small Rumin. Res. 101:55-63.

Goldhawk, C., N. Chapinal, D. M. Veira, D. M. Weary, and M. A. G. Von Keyserlingk. 2009. Prepartum feeding behavior is an early indicator of subclinical ketosis. J. Dairy Sci. 92:4971-4977.

Guard, C. L. 1994. Costs of clinical disease in dairy cows. Page 100 in Proc. Ann. Cornell Conf. Vet. Cornell Univ., Ithaca, NY.

Hernandez, F., L. Elvira, J.-V. Gonzalez-Martin, and S. Astiz. 2012. Influence of dry period length on reproductive performance and productivity of Lacaune dairy sheep under an intensive management system. J. Dairy Res. 79:352-360.

Huzzey, J. M., M. A. G. von Keyserlingk, and D. M. Weary. 2005. Changes in feeding, drinking, and standing behavior of dairy cows during the transition period. J. Dairy Sci. 88:2454-2461.

Ismail, Z. A. B., A. M. Al-Majali, F. Amireh, and O. F. Al-Rawashdeh. 2008. Metabolic profiles in goat does in late pregnancy with and without subclinical pregnancy toxemia. Vet. Clin. Pathol. $37: 434-437$.

Itle, A. J., J. M. Huzzey, D. M. Weary, and M. A. G. von Keyserlingk. 2015. Clinical ketosis and standing behavior in transition cows. J. Dairy Sci. 98:128-134.http://dx.doi.org/ 10.3168/jds.2014-7932.

Ito, K., M. A. G. von Keyserlingk, S. J. Leblanc, and D. M. Weary. 2010. Lying behavior as an indicator of lameness in dairy cows. J. Dairy Sci. 93:3553-3560.

Ito, K., D. M. Weary, and M. A. G. von Keyserlingk. 2009. Lying behavior: Assessing within- and between-herd variation in free-stallhoused dairy cows. J. Dairy Sci. 92:4412-4420.

Jolicoeur, M. S., A. F. Brito, D. E. Santschi, D. Pellerin, D. Lefebvre, R. Berthiaume, and C. L. Girard. 2014. Short dry period management improves peripartum ruminal adaptation in dairy cows. J Dairy Sci. 97:7655-7667.

Laporte-Broux, B., C. Duvaux-Ponter, S. Roussel, J. Promp, P. Chavette-Palmer, and A. A. Ponter. 2011. Restricted feeding of goats during the last third of gestation modifies both metabolic parameters and behaviour. Livest. Sci. 138:74-88.

Lima, M. S., R. A. Pascoal, G. T. Stilwell, and C. A. Hjerpe. 2012. Clinical findings, blood chemistry values, and epidemiologic data from dairy goats with pregnancy toxemia. Bovine Pract. 46:102110

Loretz, C., B. Wechsler, R. Hauser, and P. Rüsch. 2004. A comparison of space requirements of horned and hornless goats at the feed barrier and in the lying area. Appl. Anim. Behav. Sci. 87:275-283.

Malher, X., H. Seegers, and F. Beaudeau. 2001. Culling and mortality in large dairy goat herds managed under intensive conditions in western France. Livest. Prod. Sci. 71:75-86.

Marteniuk, J. V., and T. H. Herdt. 1988. Pregnancy toxaemia and ketosis of ewes and does. Vet. Clin. North Am. Food Anim. Pract. 4:307-315.

Medrano-Galarza, C., J. Gibbons, S. Wagner, A. M. De Passillé, and J. Rushen. 2012. Behavioral changes in dairy cows with mastitis. J. Dairy Sci. 95:6994-7002.

Melby, H. P., J. Aursjø, M. Binde, and H. Grøstøl. 1986. Disease in 27 Norwegian dairy goat farms. Nord. Vet. Med. 38:403-411.

Menzies, P., and D. Bailey. 1997. Diseases of the periparturient ewe. Pages 639-643 in Current Therapy in Large Animal Theriogenology. R. Youngquist, ed. WB Saunders, Philadelphia, PA.

Monzón-Gil, E., J. I. R. Castañón, and M. R. Ventura. 2010. Effect of low-forage rations on milk production of dairy goats: Separate concentrate-forage versus mixed rations. Small Rumin. Res. 94:196-200.

Navarre, C. B., and D. G. Pugh. 2002. Diseases of the gastrointestinal system. Pages 97-99 in Sheep and Goat Medicine. D. G. Pugh, ed. W. B. Saunders Co., Philadelphia, PA.

Patt, A., L. Gygax, B. Wechsler, E. Hillmann, R. Palme, and N. M. Keil. 2012. The introduction of individual goats into small estab- 
lished groups has serious negative effects on the introduced goat but not on resident goats. Appl. Anim. Behav. Sci. 138:47-59.

Patt, A., L. Gygax, B. Wechsler, E. Hillmann, R. Palme, and N. M. Keil. 2013. Factors influencing the welfare of goats in small established groups during the separation and reintegration of individuals. Appl. Anim. Behav. Sci. 144:63-72.

Pichler, M., A. Damberger, T. Arnholdt, I. Schwendenwein, J. Gasteiner, M. Drillich, and M. Iwersen. 2014. Evaluation of two electronic handheld devices for diagnosis of ketonemia and glycemia in dairy goats. J. Dairy Sci. 97:7538-7546.

Proudfoot, K. L., M. B. Jensen, P. M. H. Heegaard, and M. A. G. von Keyserlingk. 2013. Effect of moving dairy cows at different stages of labor on behavior during parturition. J. Dairy Sci. 96:16381646.

Rajala-Schultz, P. J., Y. T. Gröhn, and C. E. McCulloch. 1999. Effects of milk fever, ketosis, and lameness on milk yield in dairy cows. J. Dairy Sci. 82:288-294.

Ramin, A. G., S. Asri, and R. Majdani. 2005. Correlations among serum glucose, beta-hydroxybutyrate and urea concentrations in non-pregnant ewes. Small Rumin. Res. 57:265-269.
Rastani, R. R., R. R. Grummer, S. J. Bertics, A. Gümen, M. C. Wiltbank, D. G. Mashek, and M. C. Schwab. 2005. Reducing dry period length to simplify feeding transition cows: milk production, energy balance, and metabolic profiles. J. Dairy Sci. 88:1004-1014

Rook, J. 2000. Pregnancy toxemia of ewes, does, and beef cows. Vet. Clin. North Am. Food Anim. Pract. 16:293-317.

Schlumbohm, C., and J. Harmeyer. 2008. Twin-pregnancy increases susceptibility of ewes to hypoglycaemic stress and pregnancy toxaemia. Res. Vet. Sci. 84:286-299.

Szabò, S., K. Barth, C. Graml, A. Futschik, R. Palme, and S. Waiblinger. 2013. Introducing young dairy goats into the adult herd after parturition reduces social stress. J. Dairy Sci. 96:5644-5655. van Knegsel, A. T. M., S. G. A. van der Drift, J. Črmáková, and B. Kemp. 2013. Effects of shortening the dry period of dairy cows on milk production, energy balance, health, and fertility: A systematic review. Vet. J. 198:707-713.

Zobel, G., D. M. Weary, K. Leslie, N. Chapinal, and M. A. G. von Keyserlingk. 2015. Technical note: Validation of data loggers for recording lying behavior in dairy goats. J. Dairy Sci. 98:10821089 . 